\title{
Expanded Genetic Alphabets. Managing Nucleotides that Lack Tautomeric, Protonated, or Deprotonated Versions Complementary to Natural Nucleotides
}

Christian B. Winiger ${ }^{\mathrm{a}}$, Ryan W. Shaw ${ }^{\mathrm{a}, \mathrm{b}}$, Myong-Jung Kimª, ${ }^{\mathrm{a}, \mathrm{b}}$ Jennifer D. Moses ${ }^{\mathrm{a}, \mathrm{b}}$, Mariko F. Matsuura $^{\mathrm{a}, \mathrm{c}}$, Steven A. Benner ${ }^{\mathrm{a}, \mathrm{b}^{*}}$

Figure SI 1 Evaluation of the \% full length (FL) product formed in primer extension reactions.

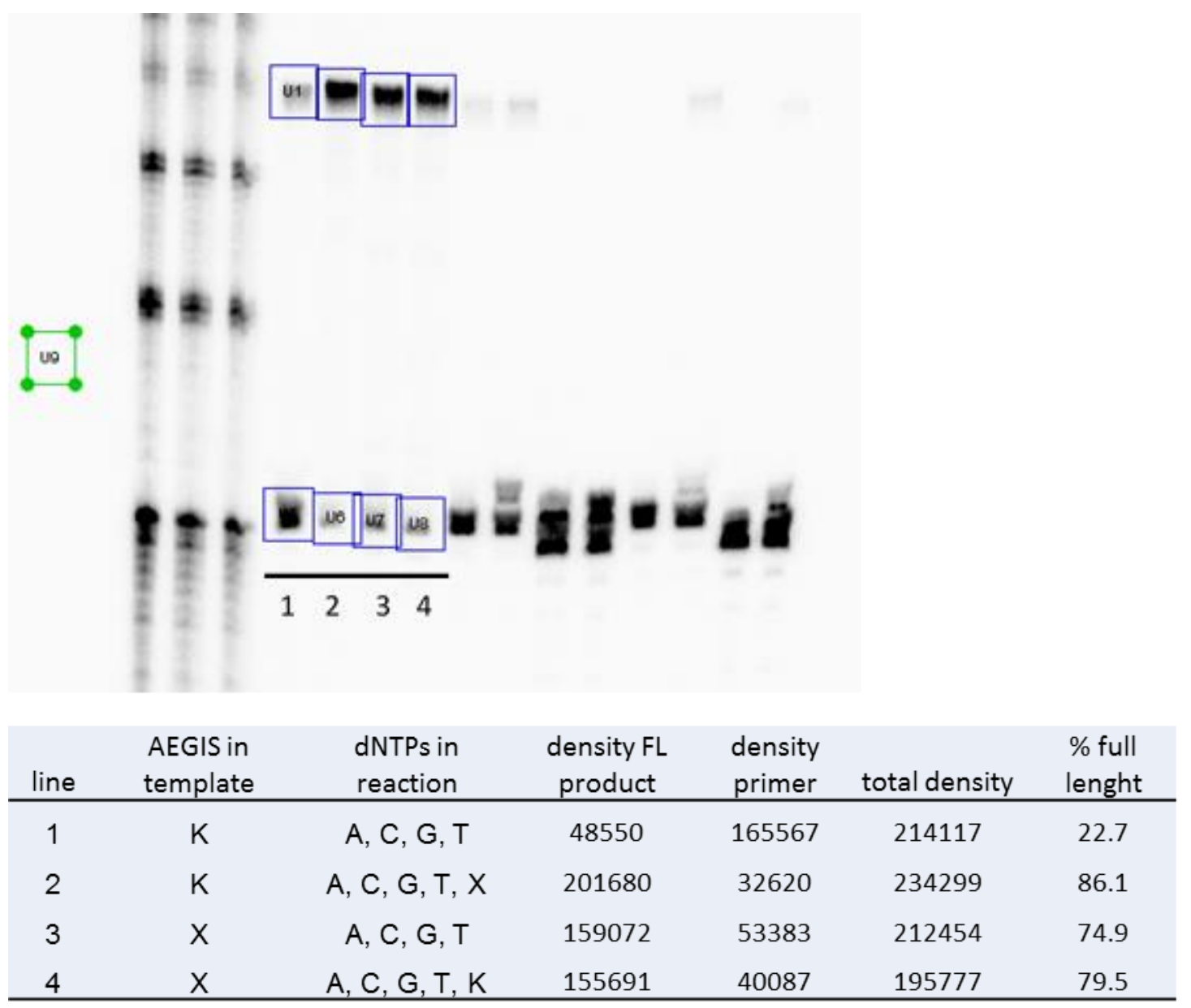

Figure SI 1 Evaluation of the \% full length product obtained in each reaction was done by subtracting the background (U9, green square) from each spot (blue squares). The sum of the total radioactivity in each line was taken as $100 \%$. Intensity of FL product was expressed in \% thereof.

Table SI I Comparing content of ligated insert in survival colonies by PCR

Survival colonies from the transformation experiments were screened by PCR to see if they contained the insert of interest. Using a $\mathrm{T}_{4}$ ligase it was found that most survival clones which should contain a double AEGIS sequence (ligation III-V) did not show the insert - rather a re-ligated vector without insert helped them to avoid to deal with the double AEGIS sequence and therefore survived. All cells that took up a proper vector with double AEGIS insert died because they were not able to read through the two AEGIS nucleotides 
or replace them properly. Using a $\mathrm{T}_{7}$ ligase the background from religated vector was reduced and a majority of the very few survival clones contained the insert.

Table SI 1 Comparison of ligations with $\mathrm{T}_{4}$ ligase and $\mathrm{T}_{7}$ ligase. Using $\mathrm{T}_{7}$ ligase reduced the background from re-ligated vector

\begin{tabular}{ccccc}
$\begin{array}{c}\text { Ligation } \\
\text { number }\end{array}$ & $\begin{array}{c}\text { AEGIS } \\
\text { sequence }\end{array}$ & $\begin{array}{c}\text { DH5 } \alpha \\
\text { T4 ligase }\end{array}$ & $\begin{array}{c}\text { DH5 } \alpha \\
\text { T7 ligase }\end{array}$ & $\begin{array}{c}\text { STL13780 } \\
\text { T7 ligase }\end{array}$ \\
\hline I & $\begin{array}{l}\text { GCCXGCC } \\
\text { CGGKCGG }\end{array}$ & $8 / 8$ & n.a. & n.a. \\
II & $\begin{array}{l}\text { GCCKGCC } \\
\text { CGGXCGG }\end{array}$ & $8 / 8$ & n.a. & n.a. \\
III & $\begin{array}{l}\text { GCCXXCCA } \\
\text { CGGKKGGT }\end{array}$ & $4 / 8$ & & $6 / 7$ \\
IV & $\begin{array}{l}\text { GCCXKCCA } \\
\text { CGGKXGGT }\end{array}$ & $0 / 8$ & $7 / 7$ & \\
V & $\begin{array}{l}\text { GGTKXACC } \\
\text { CCAXKTGG }\end{array}$ & $0 / 8$ & $4(5) / 7$ & $9 / 14$ \\
VI & $\begin{array}{l}\text { GATXTAC } \\
\text { CTAKATG }\end{array}$ & $7 / 8$ & $6 / 7$ & $5 / 7$ \\
\hline
\end{tabular}


Figure SI 2 LB agar plates with survivor clones from different sequences (Ligations I-VII with $\mathrm{T}_{7}$ ligase)

Sequences for the Ligation as in Table 1 or Table SI 1. Ligation 7 is a control ligation with only natural nucleotides in the sequence.

Top $\mathrm{DH}_{5} \alpha$ cells were plated as $60 \mu \mathrm{l}$ from a 1:10 dilution from the SOC recovery media.

Bottom STL1378o cells (MutS minus) were plated as $50 \mu \mathrm{l}$ directly from the SOC recovery media. White dots represent survivor colonies from transformation experiments. Tranformations with sequences containing double AEGIS result in only very few survivor colonies (ligation III-V).

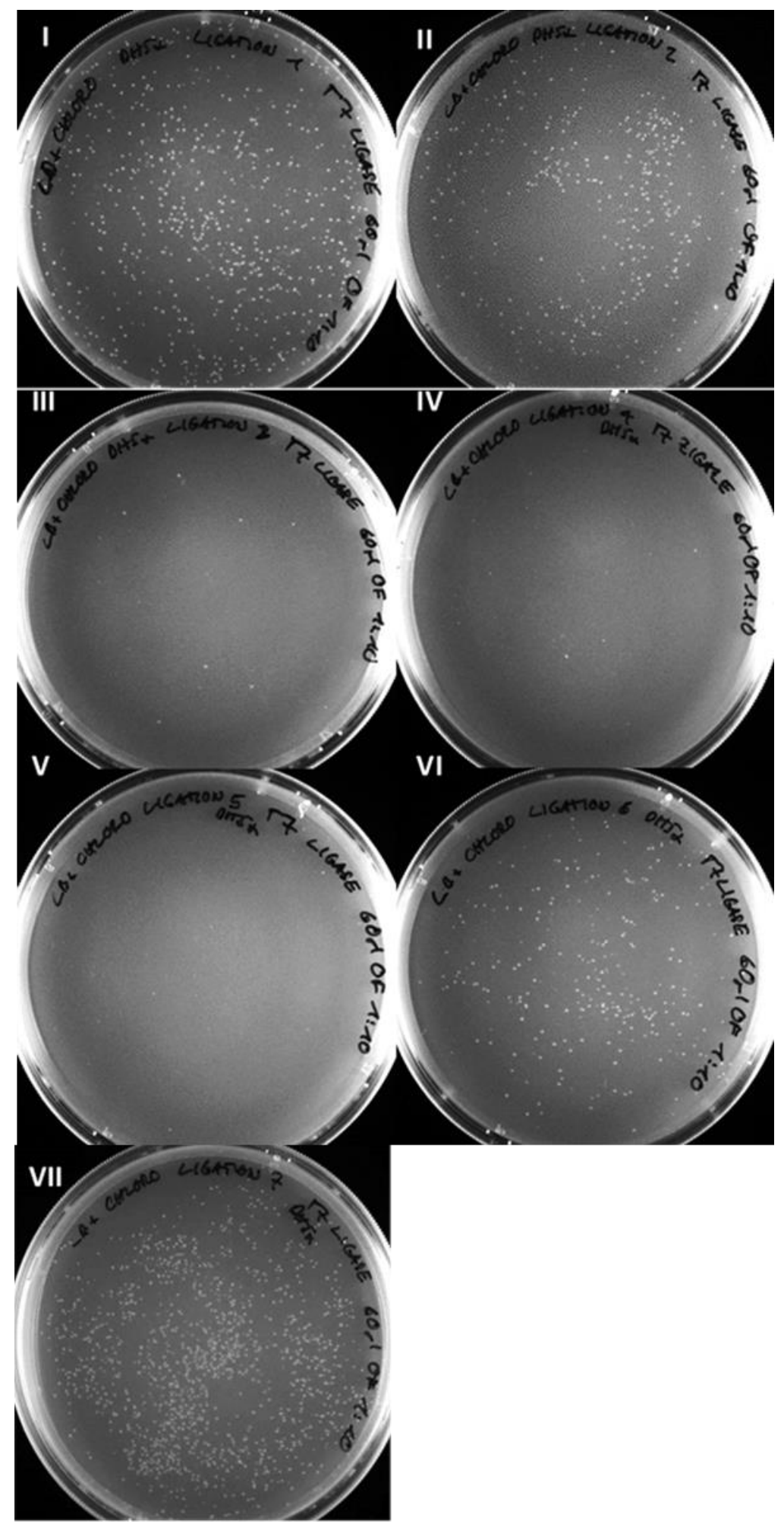


STL13780 (MutS Minus)

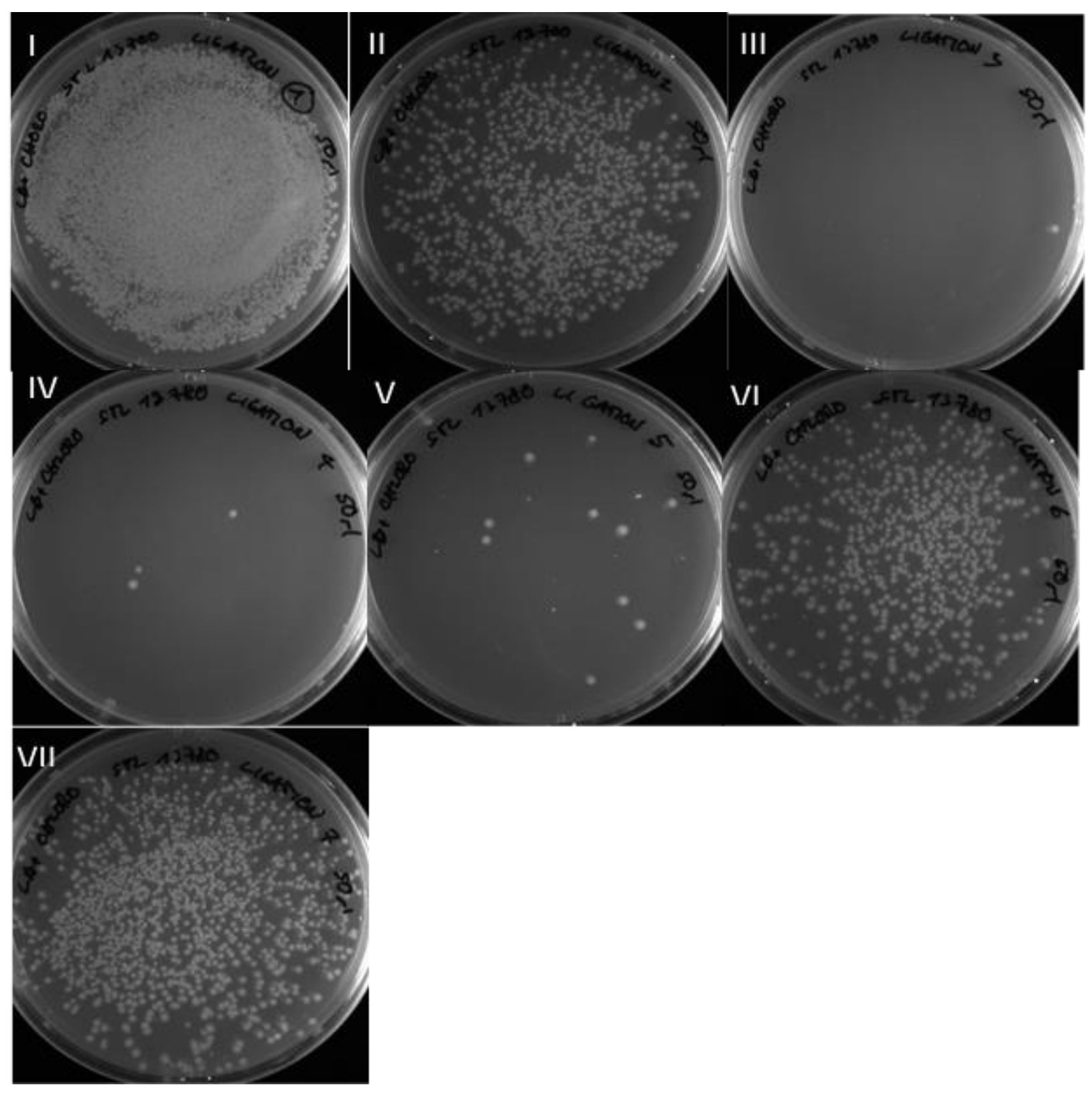




\section{Figure SI 3 Probing the mispairing between $\mathrm{dA}$ and $\mathrm{dKTP}$ and $\mathrm{dK}$ and natural nucleotides}

A 5' nuclease assay was performed to test incorporation of either dKTP opposite of dA in a template and vice versa. Assay was adapted from Wilson et al. A template containing the nucleotide to be probed (in red) and a fluor (JOE) at the $5^{\prime}$ end was annealed to a primer and a probe containing a $3^{\prime}$ quencher. The polymerase incorporates a base opposite the base to be probed, completes strand synthesis and thereby degrades the probe and releases the quencher. A fluorescent signal means successful incorporation of a base opposite the nucleotide to be probed.

Templates used were:

\section{Left:}

3' BHQ2/AGC GAG CGA GAA CGG AGC CAG GA CCG CCA CCT CCG CC 5' 5' JOE/ TCG CTC GCT CTT GCC TCG GTC CTG TTG TTA GGC GGT GGA GGC GGT 3'

\section{Right:}

3' BHQ2/CCT CAC TCA CAC TCC ACT TAC CA CCG CCA CCT CCG CC

5' JOE/ TGA GTG Agt GTG AgG TGA ATG GTG TTG TAK GGC GGT GgA GgC GG
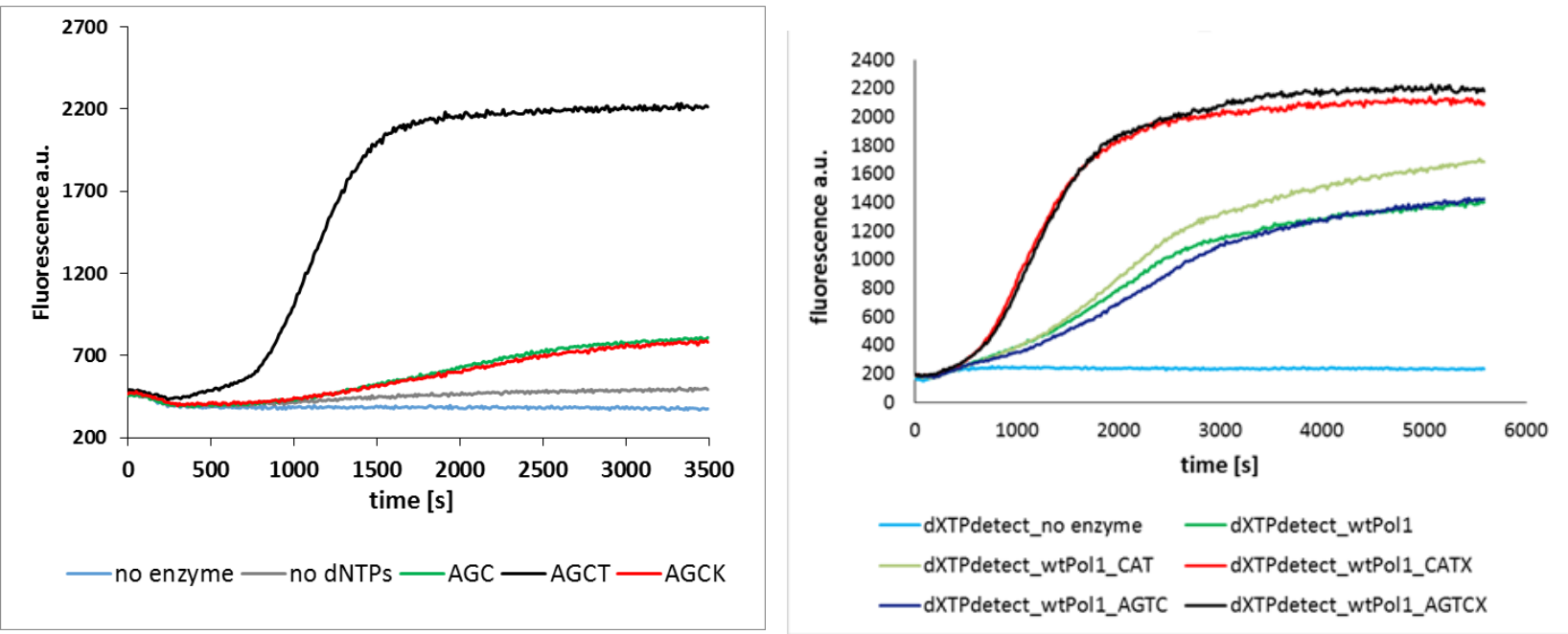

Figure SI 3 Left: incorporation of dKTP opposite of dA (see template above) is probed. Only reactions mixtures supplemented with dTTP (black line) yield full length product. No signal is seen with dKTP in the reaction mixture indicating no incorporation of dKTP opposite of dA. Right: Incorporation of dATP (or any natural bases) opposite of $\mathbf{d X}$ (see template above) is probed. Only reactions mixtures supplemented with $\mathrm{dXTP}$ (black and red line) yield full length product. No signal significantly higher than background (dark green) is seen in samples lacking dXTP. The light green curve most probably is experimental uncertainty as it contains all nucleotides also present in dark blue line - therefore should give the same intensity. 
Table SI 2 conversions of AEGIS containing sequences. Sample size from successful sequencing is indicated in parenthesis

\begin{tabular}{|c|c|c|c|c|c|}
\hline \multirow[b]{2}{*}{ Ligation \# } & \multirow[b]{2}{*}{ Sequence } & \multirow[b]{2}{*}{ what } & \multirow[b]{2}{*}{ into what } & \multicolumn{2}{|c|}{ bacterial strain } \\
\hline & & & & $\begin{array}{c}\text { in DH5a } \\
\text { (events/total } \\
\text { sequenced) } \\
\text { MutS plus }\end{array}$ & $\begin{array}{c}\text { in STL1380 } \\
\text { (events/total } \\
\text { sequenced) } \\
\text { MutS minus }\end{array}$ \\
\hline I & $\begin{array}{l}\text { GCCXGCC } \\
\text { CGGKCGG }\end{array}$ & $\mathrm{X}: \mathrm{K}$ & $\begin{array}{l}\text { A:T } \\
T: A \\
G: C\end{array}$ & $\begin{array}{c}0.909(20 / 22) \\
0.045(1 / 22) \\
0.045(1 / 22)\end{array}$ & $\begin{array}{c}1(17 / 17) \\
0 / 17 \\
0 / 17\end{array}$ \\
\hline II & $\begin{array}{l}\text { GCCKGCC } \\
\text { CGGXCGG }\end{array}$ & $\mathrm{K}: \mathrm{X}$ & $\begin{array}{l}\mathrm{T}: \mathrm{A} \\
\mathrm{C}: \mathrm{G}\end{array}$ & $\begin{array}{c}0.945(17 / 18) \\
0.055(1 / 18)\end{array}$ & $\begin{array}{c}0.9375(15 / 16) \\
0.0625(1 / 16)\end{array}$ \\
\hline III & $\begin{array}{l}\text { GCCXXCCA } \\
\text { CGGKKGGT }\end{array}$ & $X X: K K$ & $\begin{array}{l}\text { GA }: C T \\
A G: T C \\
T G: A C \\
A A: T T\end{array}$ & $\begin{array}{c}0.857(12 / 14) \\
0.0714(1 / 14) \\
0 / 14 \\
0.0711 / 14\end{array}$ & $\begin{array}{c}0.77(14 / 18) \\
0.166(3 / 18) \\
0.055(1 / 18) \\
0 / 18\end{array}$ \\
\hline IV & $\begin{array}{l}\text { GCCXKCCA } \\
\text { CGGKXGGT }\end{array}$ & XK:KX & $\begin{array}{c}\text { AC:TG } \\
\text { A:T } \\
\text { T:A } \\
\text { AA:TT } \\
\text { TC:AG } \\
\text { GC:CG }\end{array}$ & $\begin{array}{c}0.46(6 / 13) \\
0.23(3 / 13) \\
0.15(2 / 13) \\
(0 / 13) \\
0.043(1 / 13) \\
0.043(1 / 13)\end{array}$ & $\begin{array}{c}0.545(6 / 11) \\
(0 / 11) \\
0.363(4 / 11) \\
0.09(1 / 11) \\
(0 / 11) \\
(0 / 11)\end{array}$ \\
\hline V & $\begin{array}{l}\text { GGTKXACC } \\
\text { CCAXKTGG }\end{array}$ & $K X: X K$ & $\begin{array}{c}\text { CA:GT } \\
\text { A:T } \\
\text { TG:AC } \\
--- \\
-- \\
\text { T:A } \\
--G A:-C T\end{array}$ & $\begin{array}{l}0.416(5 / 12) \\
0.166(2 / 12) \\
0.083(1 / 12) \\
0.083(1 / 12) \\
0.083(1 / 12) \\
0.083(1 / 12) \\
0.083(1 / 12)\end{array}$ & $\begin{array}{c}0.455(5 / 11) \\
0.455(5 / 11) \\
0.09(1 / 11) \\
(0 / 11) \\
(0 / 11) \\
(0 / 11) \\
(0 / 11)\end{array}$ \\
\hline VI & $\begin{array}{l}\text { GATXTAC } \\
\text { CTAKATG }\end{array}$ & TXT:AKT & $\begin{array}{l}\mathrm{A}: \mathrm{T} \\
\mathrm{G}: \mathrm{C}\end{array}$ & $\begin{array}{c}0.954(21 / 22) \\
0.045(1 / 22)\end{array}$ & $\begin{array}{c}0.941(16 / 17) \\
0.059(1 / 17)\end{array}$ \\
\hline
\end{tabular}

Table SI 3 aligned raw data from sequencing

Sequences were aligned using BLAST algorithm provided by NCBI. Sequencing data for survivor clones of both transformations (STL13780 and DH5 $\alpha$ ) are shown

STL13780_Ligation I

$\begin{array}{ll}\text { Query } & 1 \\ \text { Query_169049 } & 353 \\ \text { Query_169050 } & 353 \\ \text { Query_169051 } & 354 \\ \text { Query_169052 } & 354 \\ \text { Query_169053 } & 354 \\ \text { Query_169054 } & 354 \\ \text { Query_169055 } & 354 \\ \text { Query_169056 } & 354 \\ \text { Query_169057 } & 353 \\ \text { Query_169058 } & 354 \\ \text { Query_169059 } & 353 \\ \text { Query_169060 } & 353\end{array}$

GCTCAGGTACGAGAGTCAAGGCCXGCCAGCAGTGCAGGATCAGA 44

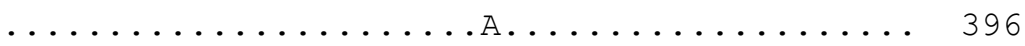

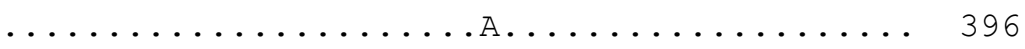

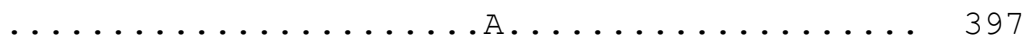

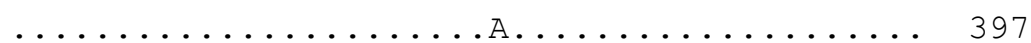

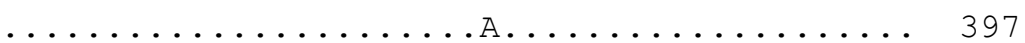

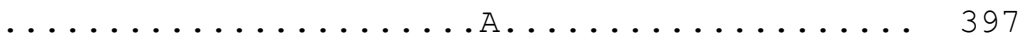

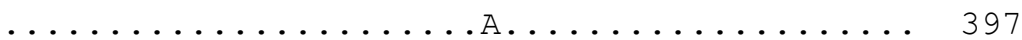

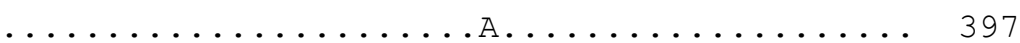

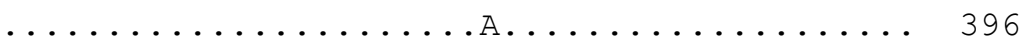

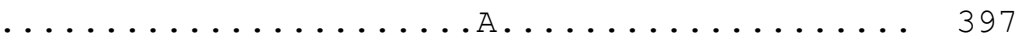

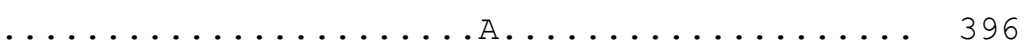

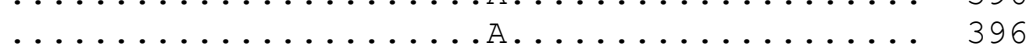


Query_169061 352

Query 169062353

Query_169063 352

Query_169064 352

Query_169065 354

\section{STL13780_Ligation II}

Query 1

Query_222852 353

Query_222853 353

Query_222854 353

Query_222855 352

Query_222856 353

Query_222857 353

Query_222859 354

Query_222860 355

Query 222862 354

Query 222863 353

Query 222864 354

Query_222865 353

Query 222866 353

Query_222867 355

Query_222868 353

Query_222869 352

\section{STL13780_Ligation III}

Query
Query_234936
Query_234937
Query_234938
Query_234939
Query_234940
Query_234941
Query_234942
Query_234943
Query_234944
Query_234945
Query_234946
Query_234947
Query_234948
Query_234949
Query_234950
Query_234953
Query_234954
Query_234955

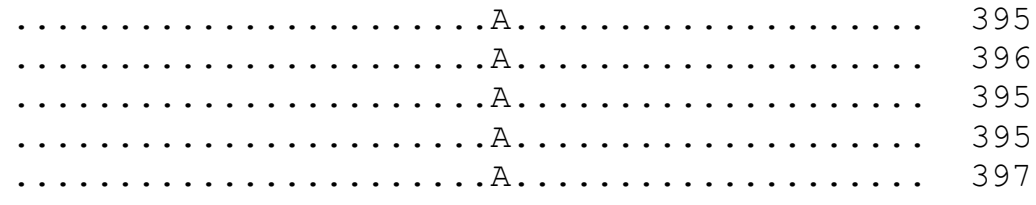

GCTCAGGTACGAGAGTCAAGGCCKGCCAGCAGTGCAGGATCAGA

44

396

396

396

395

396

396

397

398

397

396

397

396

396

398

396

395

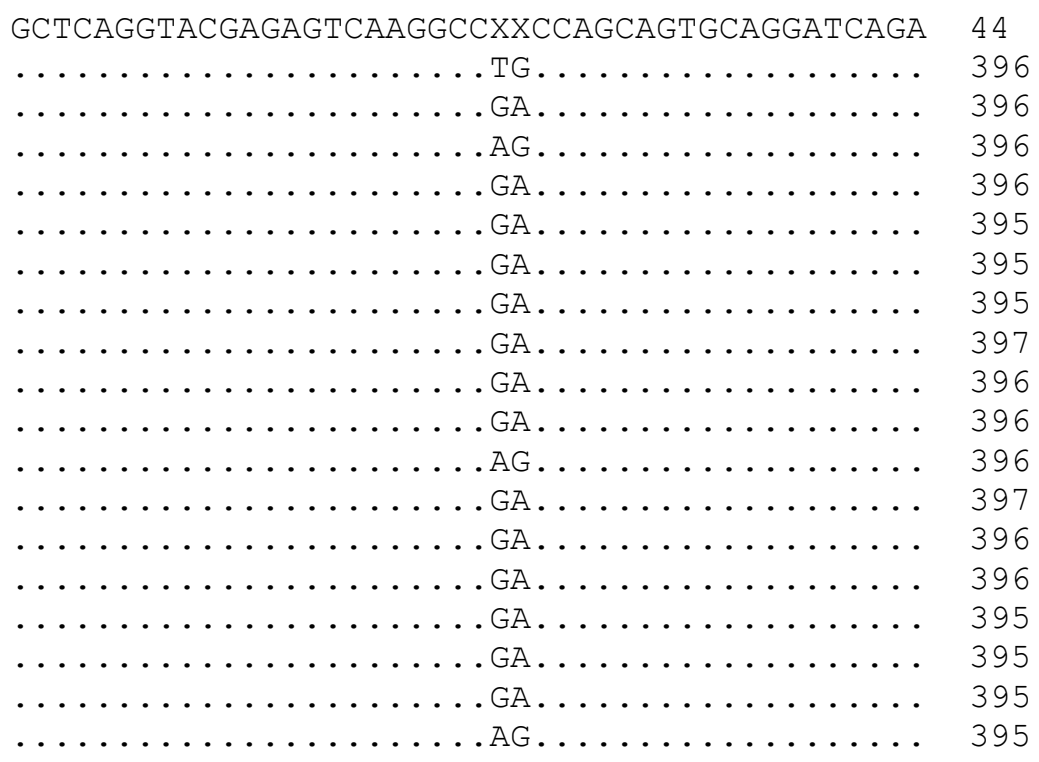

\section{STL13780_Ligation IV}

\begin{tabular}{|c|c|c|}
\hline Query & 1 & GCTCAGGTACGAGAGTCAAGGCCXKCCAGCAGTGCAGGATCAGA \\
\hline Query_115247 & 352 & $\ldots \ldots \ldots \ldots \ldots \ldots \ldots \ldots$ \\
\hline Query_115248 & 354 & 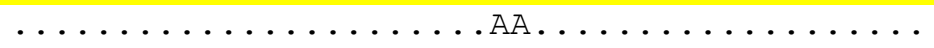 \\
\hline Query_115249 & 353 & $\ldots A C \ldots$ \\
\hline Query_115250 & 353 & 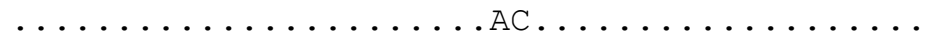 \\
\hline
\end{tabular}




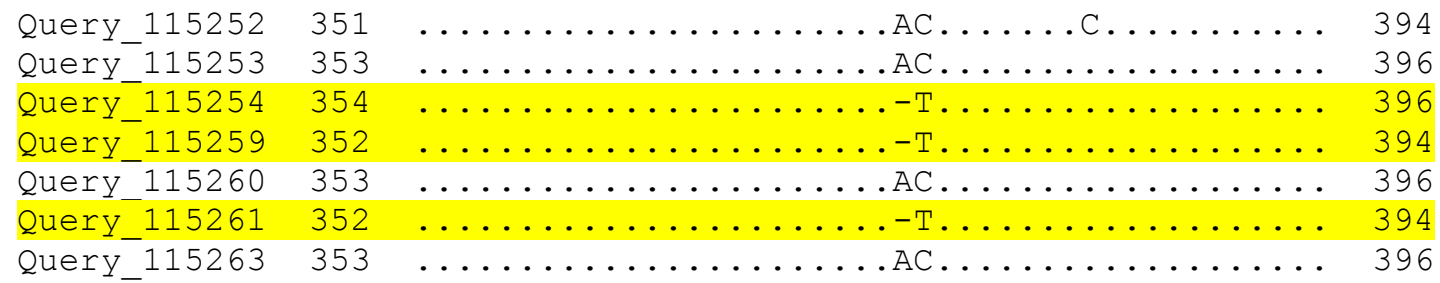

This could also be the other way round: - $\mathrm{T}$ is equal as $\mathrm{T}$ -

\section{STL13780_Ligation V}

\begin{tabular}{|c|c|c|}
\hline Query & 1 & GCTCAGGTACGAGAGTCAAGGTKXACCAGCAGTGCAGGATCAGA \\
\hline Query_22429 & 352 & $\ldots \ldots \ldots \ldots \ldots \ldots \ldots \ldots \ldots \ldots$ \\
\hline Query_22430 & 355 & 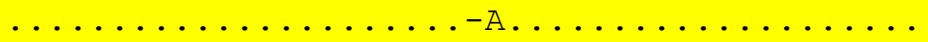 \\
\hline Query_22431 & 353 & 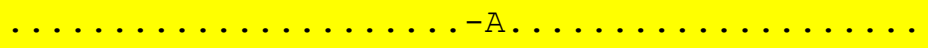 \\
\hline Query_22432 & 352 & 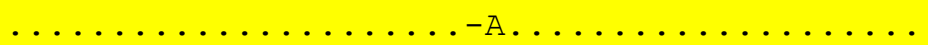 \\
\hline Query_22433 & 354 & 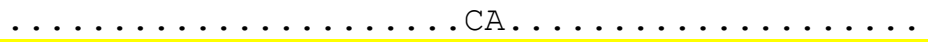 \\
\hline Query_22434 & 354 & 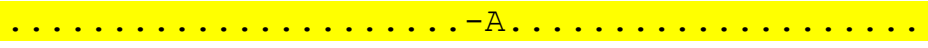 \\
\hline Query_22438 & 353 & 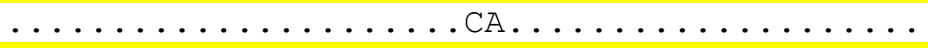 \\
\hline Query_22439 & 352 & 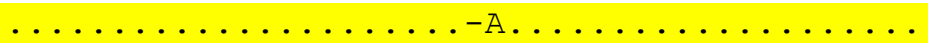 \\
\hline Query_22440 & 353 & 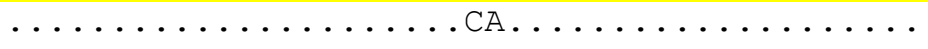 \\
\hline Query_22441 & 353 & 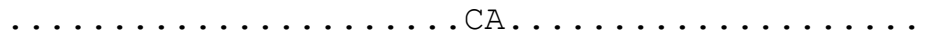 \\
\hline Query_22444 & 354 & 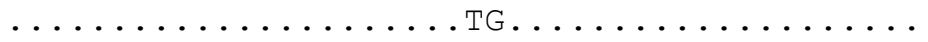 \\
\hline
\end{tabular}

This could also be the other way round: - $A$ is equal to A -

\section{STL13780_Ligation VI}

\begin{tabular}{|c|c|c|}
\hline Query & 1 & GCTCAGGTACGAGAGTCAAGGATXTACAGCAGTGCAGGATCAGA \\
\hline Query_230129 & 353 & $\ldots \ldots \ldots \ldots \ldots \ldots \ldots$ А $\ldots \ldots \ldots \ldots \ldots$ \\
\hline Query_230130 & 353 & 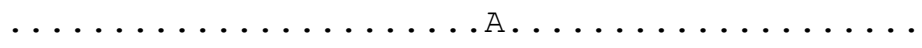 \\
\hline uery_230131 & 54 & 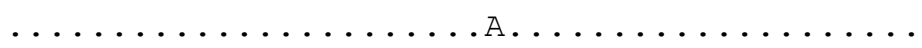 \\
\hline uery_230132 & 52 & 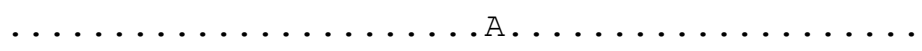 \\
\hline uery_230133 & 53 & 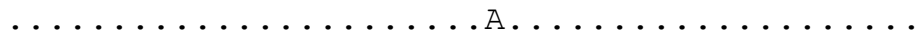 \\
\hline Query_230134 & 52 & 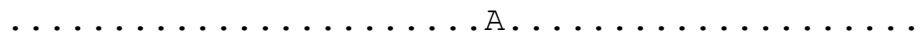 \\
\hline dery_230135 & 53 & 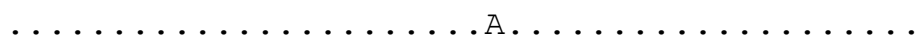 \\
\hline uery_230136 & 54 & 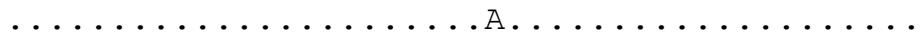 \\
\hline Query_230137 & 54 & 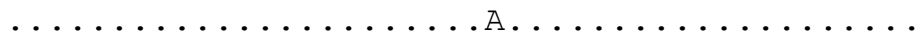 \\
\hline uery_230138 & & 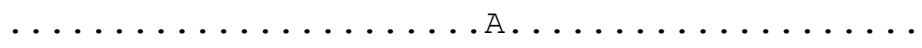 \\
\hline Query_230139 & 354 & 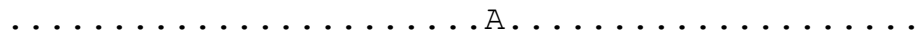 \\
\hline Query_230140 & 54 & 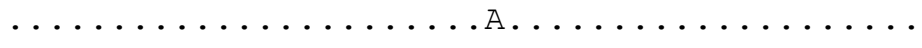 \\
\hline Query_230141 & & 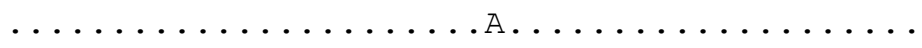 \\
\hline Query_230142 & 353 & 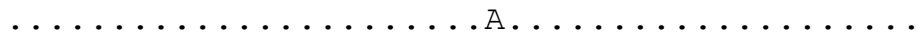 \\
\hline uery_230143 & & $\ldots \ldots \ldots \ldots \ldots \ldots \ldots \ldots \ldots$ \\
\hline Query_23 & & $\ldots \ldots \ldots \ldots \ldots \ldots \ldots \ldots \ldots \ldots \ldots \ldots \ldots$ \\
\hline Query_230145 & 53 & 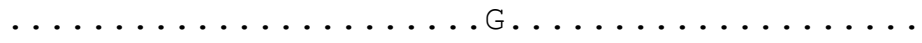 \\
\hline
\end{tabular}




\section{DH5a_ligation I}

$\begin{array}{ll}\text { Query } & 1 \\ \text { Query_184966 } & 353 \\ \text { Query_184967 } & 351 \\ \text { Query_184968 } & 353 \\ \text { Query_184969 } & 352 \\ \text { Query_184970 } & 354 \\ \text { Query_184971 } & 353 \\ \text { Query_184972 } & 352 \\ \text { Query_184973 } & 353 \\ \text { Query_184974 } & 352 \\ \text { Query_184975 } & 352 \\ \text { Query_184976 } & 354 \\ \text { Query_184977 } & 354 \\ \text { Query_184978 } & 355 \\ \text { Query_184979 } & 353 \\ \text { Query_184980 } & 352 \\ \text { Query_184981 } & 352 \\ \text { Query_184982 } & 352 \\ \text { Query_184983 } & 353 \\ \text { Query_184984 } & 354 \\ \text { Query_184985 } & 352 \\ \text { Query_184986 } & 353 \\ \text { Query_184987 } & 352\end{array}$

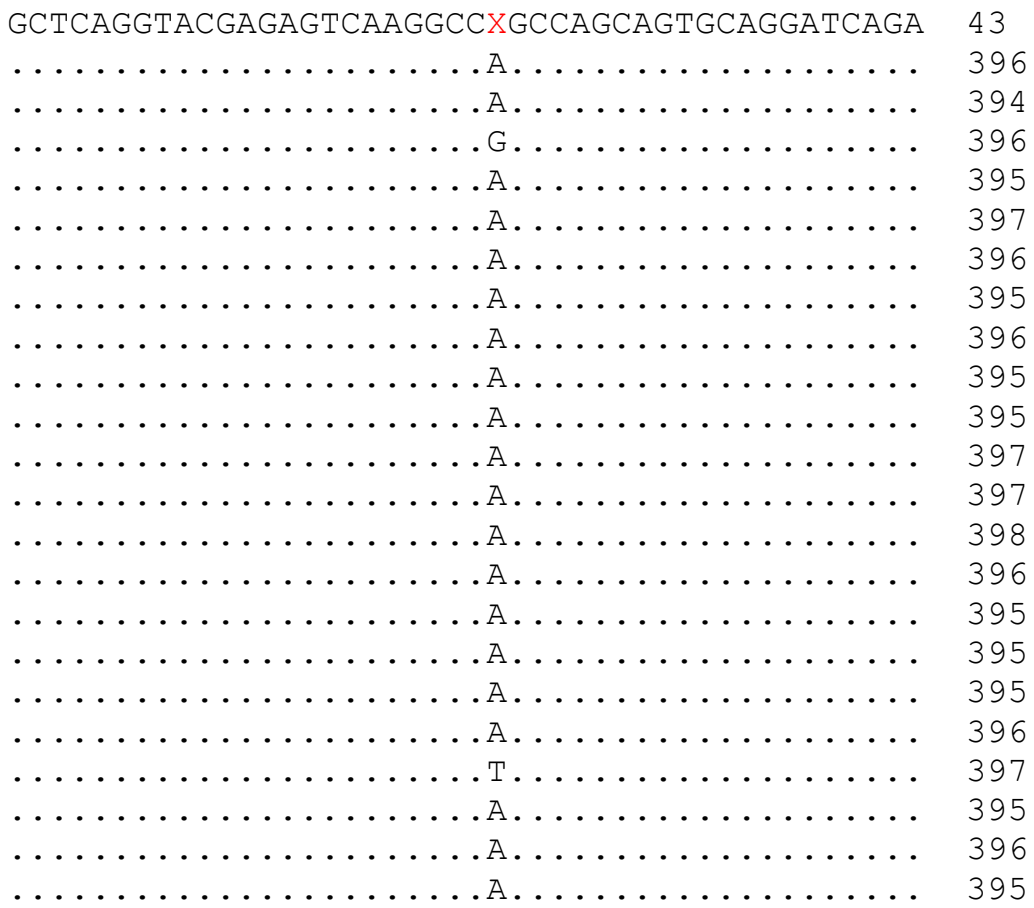

\section{DH5a_Ligation II}

$\begin{array}{ll}\text { Query } & 1 \\ \text { Query_66551 } & 357 \\ \text { Query_66552 } & 353 \\ \text { Query_66553 } & 352 \\ \text { Query_66554 } & 354 \\ \text { Query_66555 } & 354 \\ \text { Query_66556 } & 352 \\ \text { Query_66557 } & 352 \\ \text { Query_66558 } & 353 \\ \text { Query_66559 } & 354 \\ \text { Query_66560 } & 352 \\ \text { Query_66561 } & 354 \\ \text { Query_66562 } & 351 \\ \text { Query_66563 } & 353 \\ \text { Query_66564 } & 352 \\ \text { Query_66565 } & 351 \\ \text { Query_66566 } & 353 \\ \text { Query_66567 } & 353 \\ \text { Query_66568 } & 352 \\ \text { Query_66569 } & 352\end{array}$

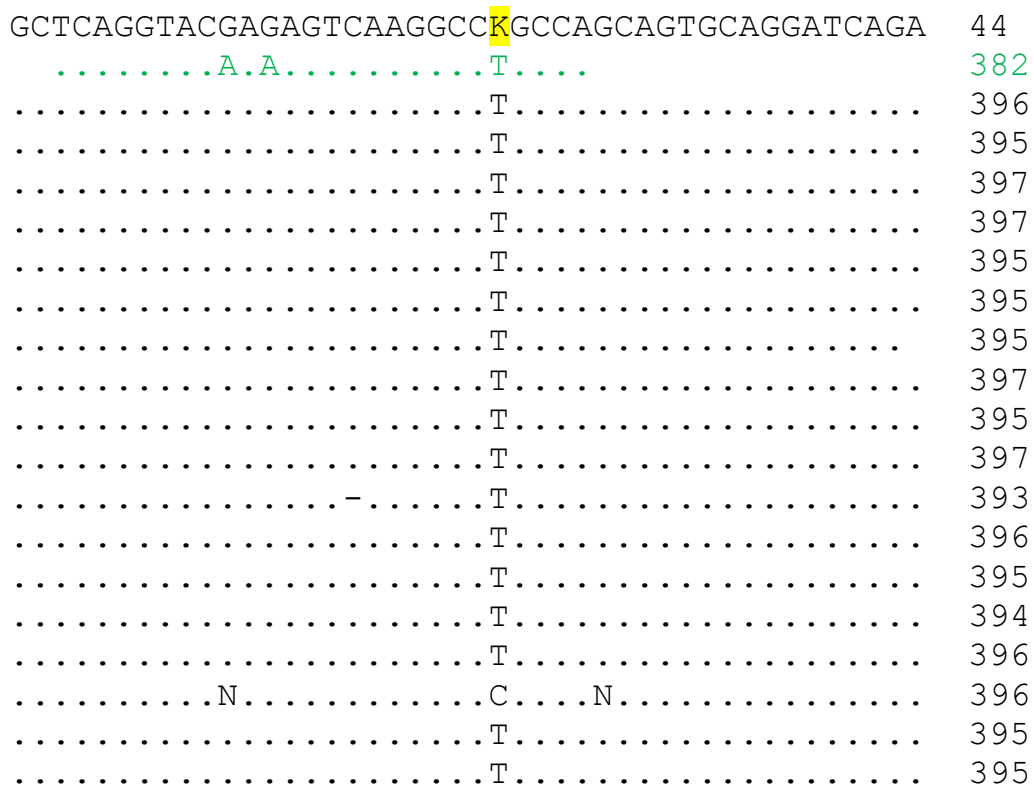

DH5a_Ligation III

\begin{tabular}{|c|c|c|}
\hline Query & 1 & GCTCAGGTACGAGAGTCAAGGCCXXCCAGCAGTGCAGGATCAGA \\
\hline Query_159091 & 353 & 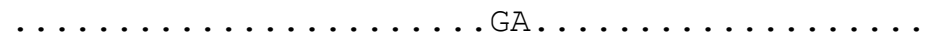 \\
\hline Query_159092 & 353 & $\ldots \ldots \ldots \ldots \ldots \ldots \ldots \ldots \ldots \ldots$ \\
\hline Query_15 & 353 & $\ldots$ GA $\ldots$ \\
\hline
\end{tabular}




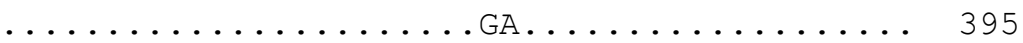

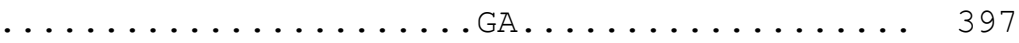

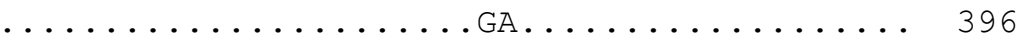

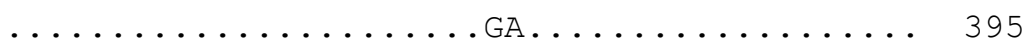

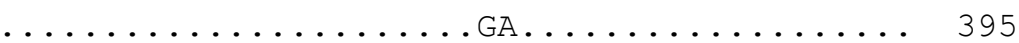

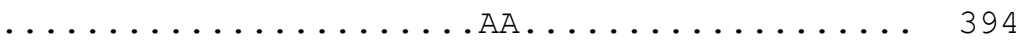

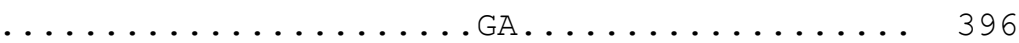

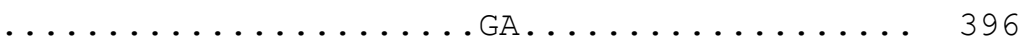

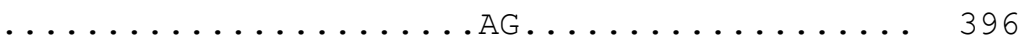

\section{DH5a_Ligation IV}

\begin{tabular}{|c|c|c|c|}
\hline & & CTCAGGTACGAGAGTCAAGGCCXKCCAGCAGTGCAGGATCAGA & \\
\hline Query_115510 & 353 & 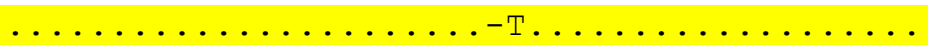 & \\
\hline uery_115511 & 52 & 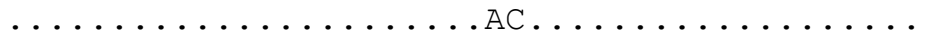 & \\
\hline dery_115512 & 52 & 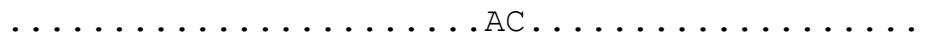 & \\
\hline uery_11 & & 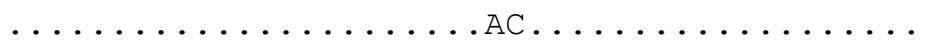 & \\
\hline Y_11 & 53 & $\ldots \ldots \ldots \ldots \ldots \ldots \ldots \ldots \ldots \ldots$ & \\
\hline Y_11 & 52 & $\ldots \ldots \ldots \ldots \ldots \ldots \ldots \ldots \ldots \ldots \ldots \ldots \ldots \ldots \ldots \ldots$ & \\
\hline$y_{-} 1$ & 2 & $\ldots \ldots \ldots \ldots \ldots \ldots \ldots \ldots+\ldots \ldots \ldots \ldots \ldots$ & \\
\hline uery_11 & 53 & 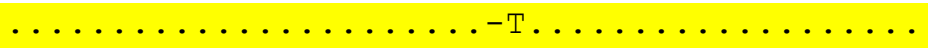 & \\
\hline y_11 & & $\ldots \ldots-A \ldots \ldots \ldots \ldots \ldots$ & \\
\hline uery_11 & & $\ldots \ldots-A \ldots \ldots \ldots \ldots \ldots \ldots$ & \\
\hline uery_115 & & $\ldots \ldots A C \ldots \ldots \ldots \ldots \ldots$ & \\
\hline ery_115 & & $\ldots-$ A.... & \\
\hline uery_115525 & 53 & $\ldots \ldots \ldots \ldots \ldots \ldots$ AC $\ldots \ldots$ & \\
\hline
\end{tabular}

\section{Could also be vice versa:}

\section{DH5a Ligation V}

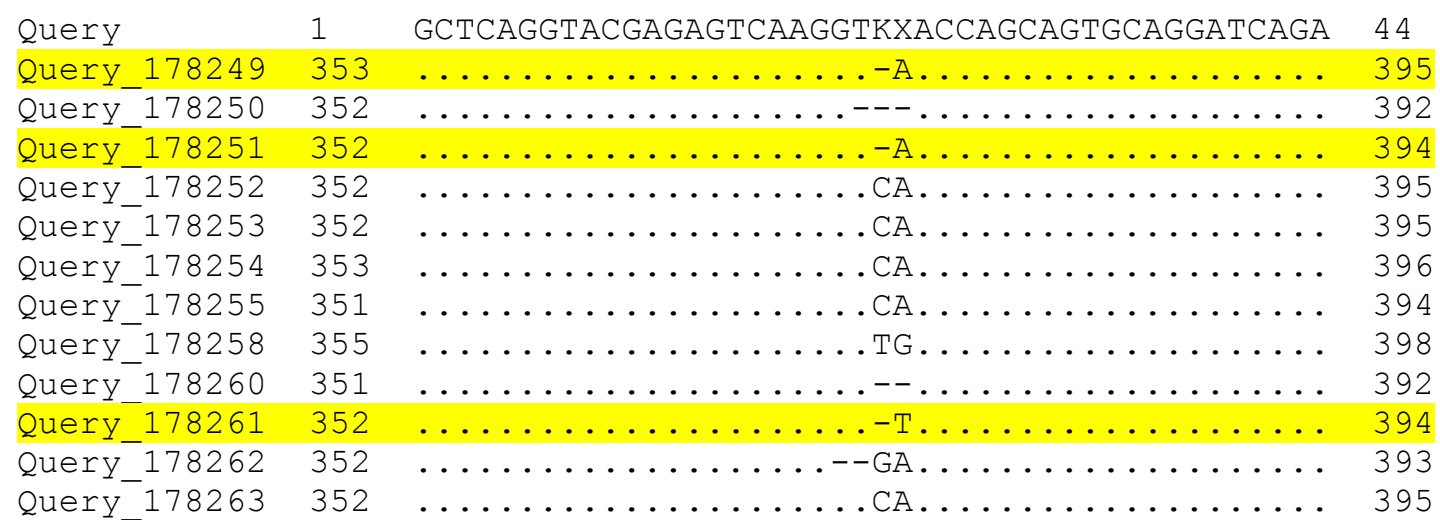

Could also be opposite: $-T$ is equal to $T-$ and $-A$ is equal to $A$ - 


\section{DH5a_Ligation VI}

\begin{tabular}{|c|c|c|}
\hline Query & 1 & GCTCAGGTACGAGAGTCAAGGATXTACAGCAGTGCAGGATCAGA \\
\hline Query_48663 & 353 & 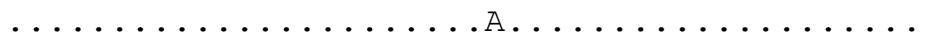 \\
\hline Query_48664 & 353 & 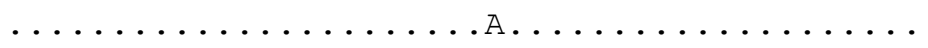 \\
\hline Query_48665 & 356 & 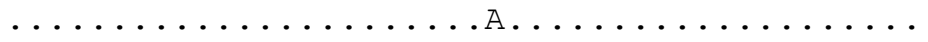 \\
\hline Query_48666 & 351 & 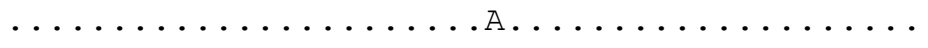 \\
\hline uery_48667 & 353 & 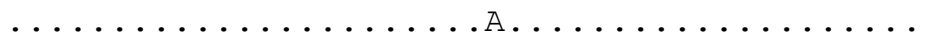 \\
\hline Query_48668 & 353 & 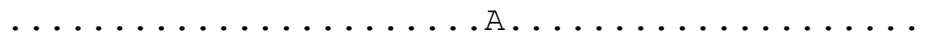 \\
\hline uery_48669 & 353 & 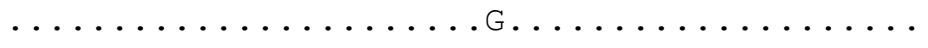 \\
\hline uery_48671 & 353 & 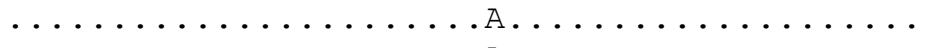 \\
\hline Query_48672 & 354 & 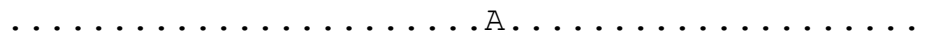 \\
\hline Query_48673 & 354 & 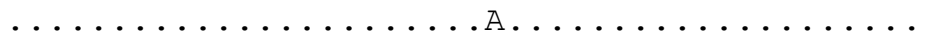 \\
\hline Query_48674 & 54 & $\cdots \cdots \cdots \cdots \cdots \cdots \cdots \cdots \cdots \cdots$ \\
\hline Query_48675 & 355 & 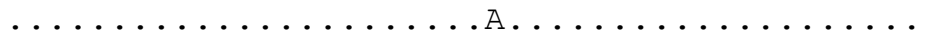 \\
\hline Query_48676 & 362 & $\ldots \ldots \ldots \ldots \ldots \ldots \ldots \ldots$ \\
\hline Query_48677 & 352 & 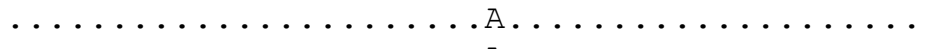 \\
\hline Query_48678 & 353 & $\cdots \cdots \cdots \cdots \cdots \cdots \cdots \cdots \cdots$ \\
\hline Query_48679 & 354 & 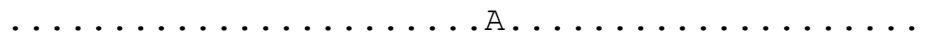 \\
\hline Query_48680 & 353 & $\cdots \cdots \cdots \cdots \cdots \cdots \cdots \cdots \cdots \cdots \cdots \cdots$ \\
\hline Query_48681 & 355 & $\cdots \cdots \cdots \cdots \cdots \cdots \cdots \cdots \cdots$ \\
\hline Query_48682 & 354 & 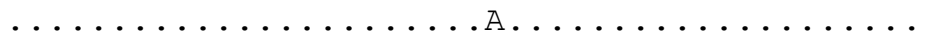 \\
\hline Query_48683 & 54 & 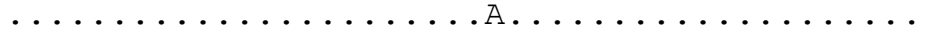 \\
\hline uery_48 & & $\cdots \ldots \ldots \ldots \ldots$ \\
\hline uery_486 & 52 & 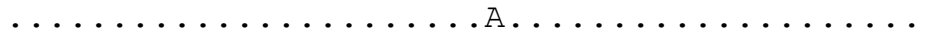 \\
\hline
\end{tabular}

\section{SI Figure 4 Quality control for AEGIS containing inserts}

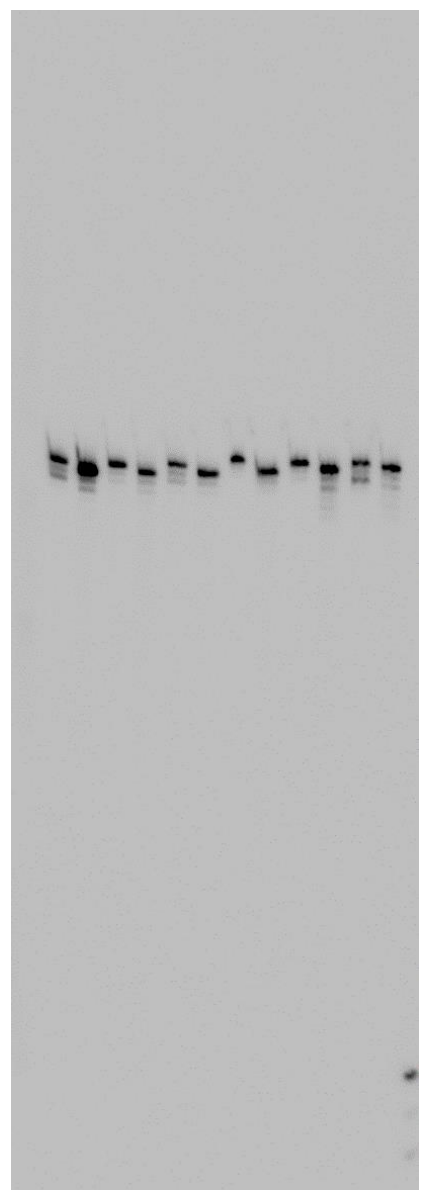

Figure SI 4 Page purified inserts were PAGE purified and 5' radiolabelled for quality control. Sequences show alternating $45 \mathrm{bp}$ and $44 \mathrm{bp}$. 